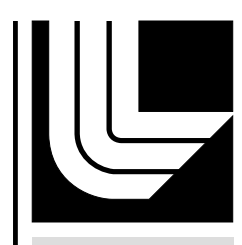

LA W REN CE LIVERM ORE NATIONAL LABORATORY

Fission TPC Beam Time Needs

M. Heffner

October 4, 2012 
This document was prepared as an account of work sponsored by an agency of the United States government. Neither the United States government nor Lawrence Livermore National Security, LLC, nor any of their employees makes any warranty, expressed or implied, or assumes any legal liability or responsibility for the accuracy, completeness, or usefulness of any information, apparatus, product, or process disclosed, or represents that its use would not infringe privately owned rights. Reference herein to any specific commercial product, process, or service by trade name, trademark, manufacturer, or otherwise does not necessarily constitute or imply its endorsement, recommendation, or favoring by the United States government or Lawrence Livermore National Security, LLC. The views and opinions of authors expressed herein do not necessarily state or reflect those of the United States government or Lawrence Livermore National Security, LLC, and shall not be used for advertising or product endorsement purposes.

This work performed under the auspices of the U.S. Department of Energy by Lawrence Livermore National Laboratory under Contract DE-AC52-07NA27344. 


\section{Fission TPC Beam Time Needs}

October 5, 2011

Document version 1.0 


\section{Contents}

1 Introduction $\quad 2$

2 Systematic Uncertainties in Fission Cross Section Measurements 3

3 Experimental Configurations $\quad 7$

3.1 Example Calculation of Required Number of Neutrons . . . . . . . . . . 7

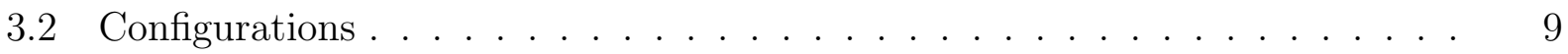

4 Beam Estimates $\quad 9$

4.1 LANSCE . . . . . . . . . . . . . . . . . . . . . . . 10

4.2 Fixed energy source . . . . . . . . . . . . . . . . . 11

5 Conclusion $\quad 11$

\section{Introduction}

The TPC project is being funded to deliver fission data of unprecedented precision and is supported by DOE through NNSA and NE programs. The goal of measuring sub-percent fission cross sections is a challenge that requires a technique that provides the level of detail necessary to understand and minimize the variety of systematic uncertainties that have plagued these measurements in the past. Vast amounts of $\mathrm{Pu}-239$ fission cross section data have been collected in a wide variety of experiments over the last 60 years and the remaining uncertainties in the cross section remain around 2-3\% and are entirely dictated by the systematic uncertainties. Any single sub-percent fission cross section measurement will need to convincingly demonstrate that the dominating systematic uncertainties are understood their assignments meaningful. The Time Projection Chamber is a tool that should provide all the necessary insights in understanding and addressing all of the largest known (and potentially unknown) systematic uncertainties in these types of measurements. The primary objective is to deliver high precision fission cross section data on the major actinides $\mathrm{Pu}-239$, U-235 and U-238 with a longer term program envisioned to support not only follow-on application specific measurements but a more comprehensive science-based program to address the needs of the theorists and modelers to deliver higher precision, fully covariant evaluations for the full suite of actinides. The TPC time line for the next 4 years is driven by the NNSA need to have the new $\mathrm{Pu}-239$ fission cross section data available to applications by the beginning of FY16, with an intermediate $\mathrm{Pu}-239 / \mathrm{U}-235$ precision fission ratio measurement delivery in FY14. Figure 1 shows the current projected time line for the experiment.

The project has moved forward in an evolutionary and prudent fashion to ensure that technological choices were not made too early and that enough testing could be completed to succeed at the overall project while maintaining an aggressive 4 year delivery schedule. Development of the full potential of the TPC as a research tool to address the broader science needs could easily be a 20-year program, but the near term is driven by a ranked list of fission cross section measurement systematic uncertainties, which are detailed in the first section. The second section is a more detailed discussion of the specific configurations and 


\section{TPC Project Timeline}
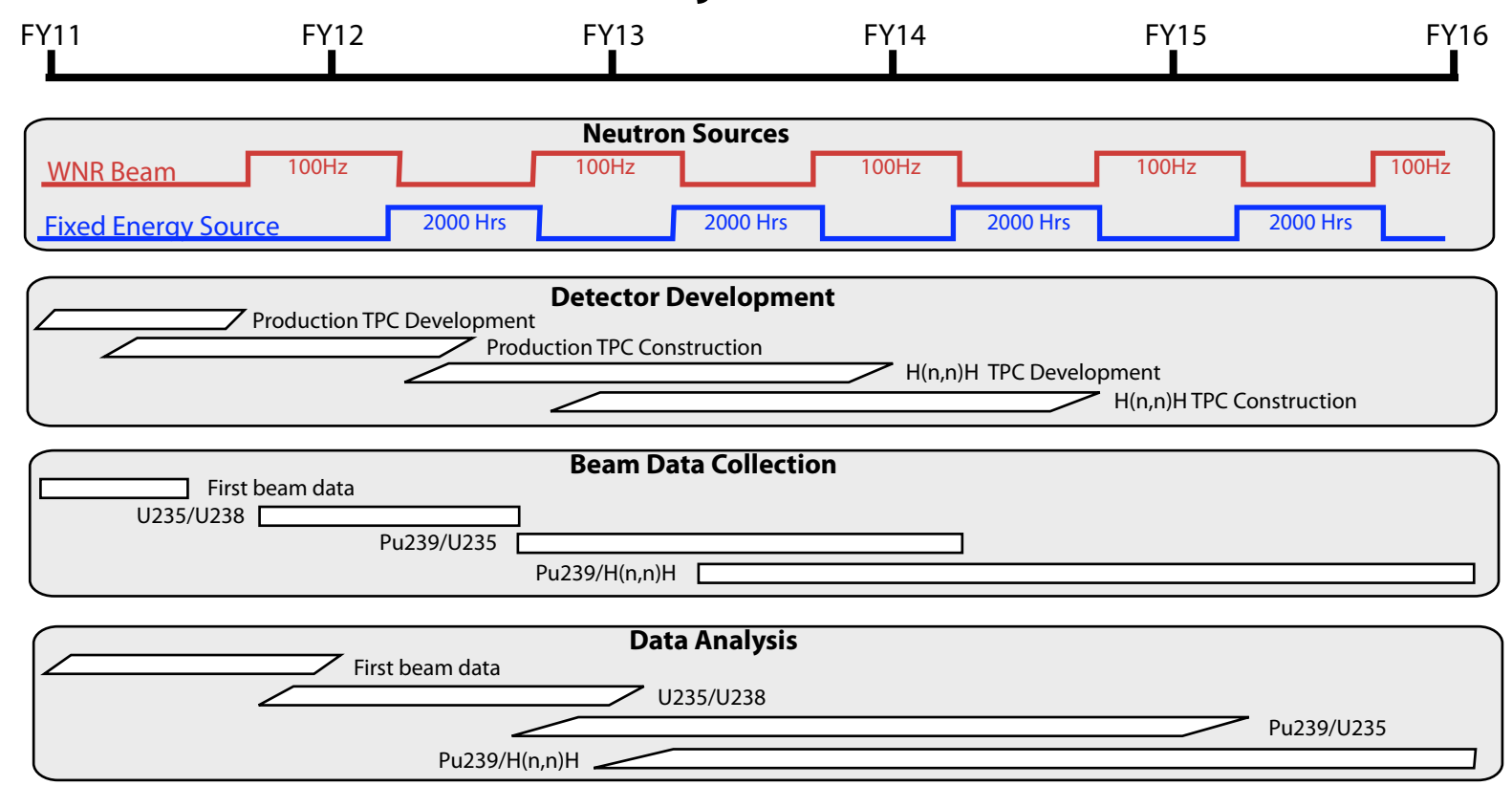

\section{Major Milestones/Deliverables Timeline}

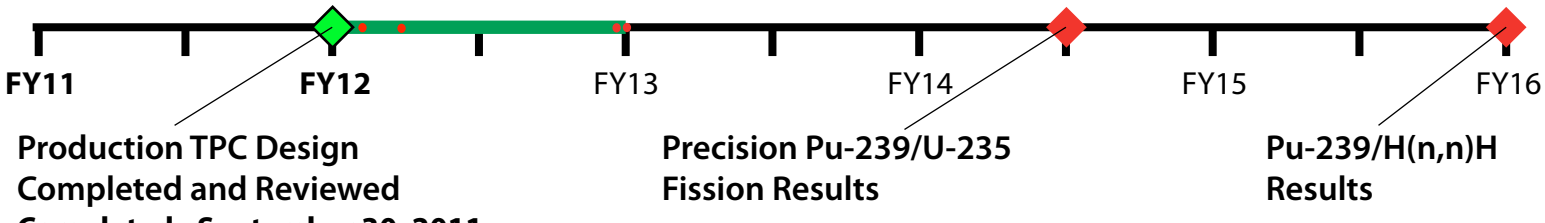

Completed - September 30, 2011

FY12 Supporting Milestones/Deliverables

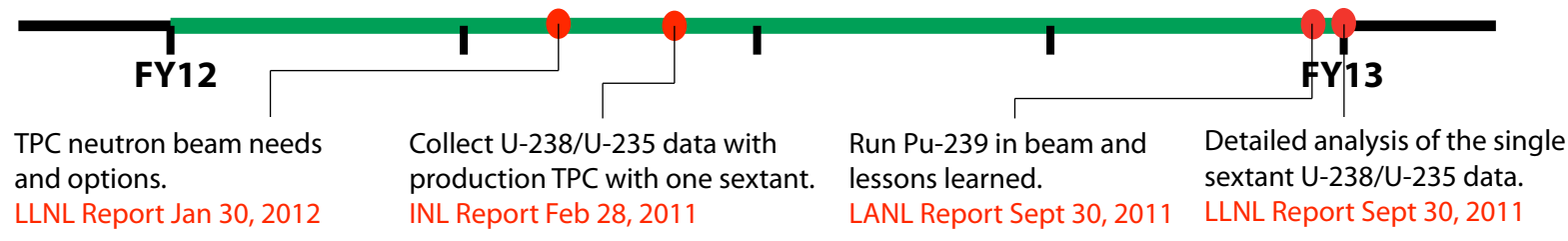

Figure 1: The current timeline for the TPC experiment to measure the $\mathrm{Pu}-239$ cross section

statistics required to minimize the uncertainties with the largest impact. The last section is a discussion of the neutron beams estimates required to meet the milestones.

\section{Systematic Uncertainties in Fission Cross Section Mea- surements}

Cross section measurements are simple counting experiments that are complicated by the realities of the physics, the experimental environment and the available tools. Arriving at an 
absolute neutron induced fission cross section, one must count:

- the total number or flux of projectiles (neutrons),

- the total number of target nuclei in the problem $(\mathrm{Pu}-239)$,

- the total number or rate of fission events.

Table 1 contains an extensive list of sources of uncertainties encountered in neutron beam experiments. The list was developed during the proposal stage of the TPC project. Input was provided by researchers who have performed a variety of similar measurements at a number of facilities.

\begin{tabular}{|l|l|l|}
\hline \multirow{2}{*}{ Source of Uncertainty } & \multicolumn{2}{|c|}{ Estimated Uncertainty } \\
\cline { 2 - 3 } & Fission Chamber & TPC \\
\hline \hline Total & $\mathbf{1 . 6 2 \%}+\mathbf{?} ?$ & $\mathbf{0 . 4 8 \%}$ \\
\hline \hline Neutron Beam & $0.10 \%$ & $0.10 \%$ \\
\hline Neutron Energy TOF & $1.00 \%$ & $0.40 \%$ \\
\hline Flux Measurement, ${ }^{235}$ U vs. $\mathrm{H}_{2}$ ref & $?$ & Very Small \\
\hline Beam Profile & $?$ & Very Small \\
\hline Energy Position Dependence & $?$ & Very Small \\
\hline Beam Flux Outside Target & $?$ & Very Small \\
\hline Beam Spreading and Attenuation & $0.30 \%$ & $0.10 \%$ \\
\hline \hline Target & $0.10 \%$ & Very Small \\
\hline Purity & $?$ & $0.10 \%$ \\
\hline Surface Contamination & $0.70 \%$ & $0.05 \%$ \\
\hline Non-uniform Target Density & $0.10 \%$ & $0.20 \%$ \\
\hline Energy Loss in Target, 2 components: \\
\hline 1. Complete Loss & $1.0 \%$ & $0.00 \%$ \\
\hline 2. Particle ID Degradation & $0.00 \%$ \\
\hline \hline Fragments & $0.00 \%$ & \\
\hline Partial Containment Track & $?$ & \\
\hline Pulse Height Variation &
\end{tabular}

Table 1: Summary of systematic errors to be studied with the TPC. The items in red have the largest effect on the final result. Total error estimated by a quadrature sum of the individual errors. The "?" means that the error is difficult to quantify in the case of the fission chamber. The "Very Small" comment means that it should be negligible for the current goal. Because the error on the ${ }^{239} \mathrm{Pu}$ cross section is of order $2-3 \%$ and we estimate the known errors at $1.62 \%$ some additional error is hidden in the items with a "?" and will be studied with the TPC measurements.

Experimental neutron beams typically have non-uniform profiles with non-uniform energy distributions across the profile. The neutron energy spectrum itself has a shape that is driven by the type of source, the collimation system used and all subsequent scattering, leading 
halos, attenuation and spreading of the beam. The ability to reconstruct charged particle trajectories in the TPC will provide highly refined spatial information about variations in the physical beam characteristics, which are typically ignored in fission ratio measurements. These variations, combined with non-uniformities in target materials, lead to a bias in a measurement. These types of biases will be removed in the TPC experiments given the 3D spatial reconstruction capabilities. The largest sources of uncertainties are in determining the total number of neutrons or flux. The most precise measurements in the past have used parallel plate ionization chambers where one set of plates counts fission events of a target material and the other plates count the fission events from a standard material, such as U-235. In these experiments, the beam is counted by the U- 235 foil and the argument is that most of the other beam related systematic uncertainties are minimized in this ratio configuration, except for the beam spreading and attenuation effects, which have to be calculated, and the normalization liability, which is about $1 \%$ for $\mathrm{U}-235$ in the fast region. Figure 2 is a plot of the time of flight measured for a U-238 target at the WNR facility. The TOF is converted to an incident neutron energy given the known flight path length and mass of the neutron. The neutron beam does not have a uniform energy distribution, which is true at all neutron facilities, at a minimum, given the very nature of neutrons. There are further complications due to specific beam delivery systems. The flux must be understood and measured as a function of energy to insure the cross section measurement is unbiased by the neutron energy distribution and facility artifacts.

The hydrogen TPC measurement will replace the U-235 flux normalization with hydrogen elastic scattering, which is known to almost $0.2 \%$ as a function of incident neutron energy. This will remove the $1 \%$ liability of using the U-235 fission cross section and provide scattering beam diagnostics to minimize the previously unmeasured scattering and attenuation contributions.

The targets that will be used in the TPC experiments are also not perfect. Much work has gone into minimizing the backing material and fabricating a uniform target area. The stock material purchased for the $\mathrm{Pu}-239$ targets is of high purity but energy dependent corrections will also be made to remove the bias from the primary contaminant, $\mathrm{Pu}-240$. The uniformity of experimental targets is typically the largest source of uncertainty. Highly efficient evaporative techniques have been developed in this project to minimize the variations in the thin deposits to insure uniform transmission of fission and alpha particles. Given the power of the TPC track reconstruction capabilities, the liability of variations in thickness is minimized since the origin of the alphas (radioactive decay) and fission products can be measured to within a few tens of microns, the isotropy assumption usually present is removed and variations can be accounted for, even when folded with non-isotropic beams. The total mass normalization will come from the auto-radiograph of the $\mathrm{Pu}-239$ target inside the TPC while it is in the beam or out.

Although fission fragments and lighter particles, like hydrogen or alphas, are relatively easy to distinguish in most cases by their specific ionization in a gaseous detector, an overlap develops between the two ideally separated distributions due to scattering in the target material and backing. Energy degraded fission fragments will occasionally deposit a total energy less than a full energy alpha. Without any further discrimination, this results in a counting ambiguity systematic uncertainty of order 1\%. A simple simulation makes this point very clear (see figure 3). 


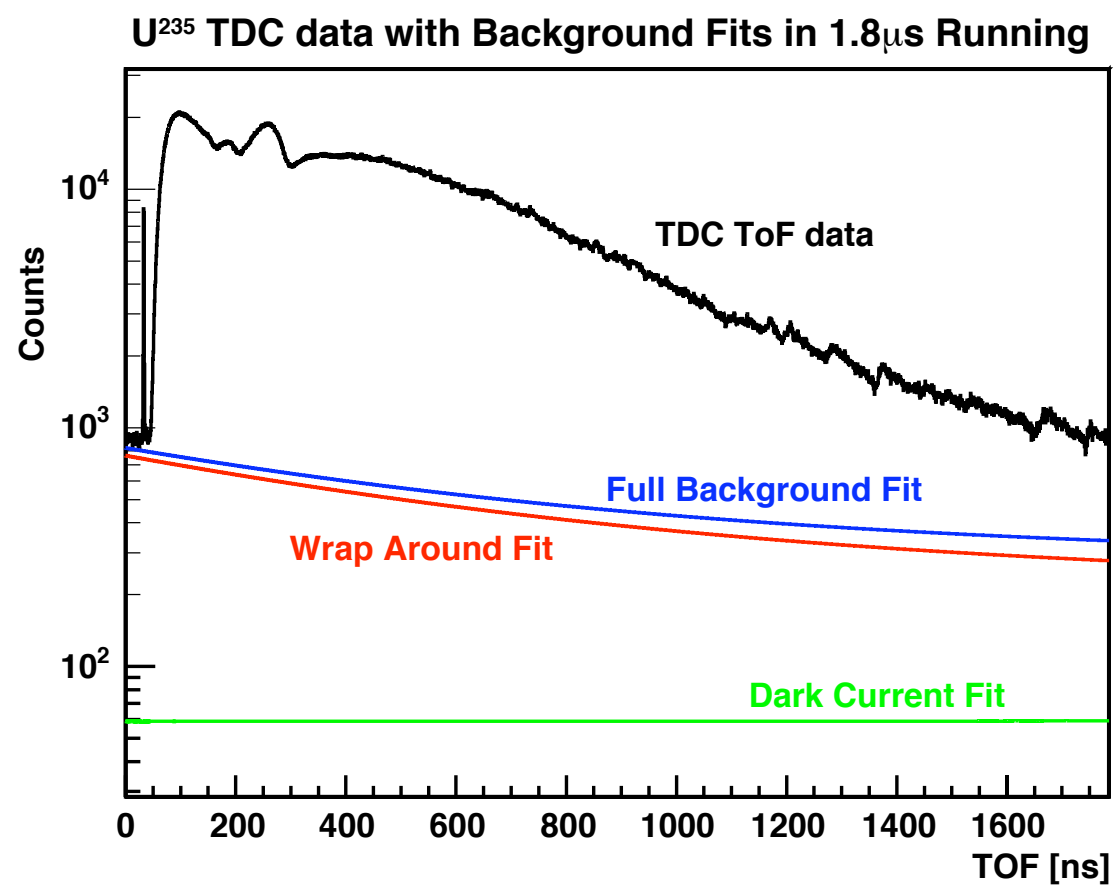

Figure 2: Shown here is a Time of Flight distribution based on fission events in U235 as measured at 90L at the LANSCE WNR facility. The ToF can be converted from time to incident neutron energy. The lower times are fast arriving neutrons (high energy) and the longer times are slower to arrive neutrons (low energy). Facility artifacts, such as accelerator dark current and frame overlap (wrap around), are also shown.

The ability of the TPC to reconstruct the track, and most simply, its length, provides an extra dimension for separating charged particle species. Figure 4 is a plot showing the track length as a function of the measured track energy. This simple metric is enough to remove the $1 \%$ uncertainty in typical fission experiments. Of course, the specific ionization along the track provides even higher fidelity information and potentially useful fission fragment identification but the focus early in the project is to remove the primary uncertainty from particle identification and insure that a subpercent fission cross section ratio is attainable. Recent results from operating a full sextant has confirmed this performance metric.

The TPC has been designed to significantly reduce the systematic uncertainties in neutron induced fission cross section measurements and addresses all the major contributors to a level such that a sub-percent measurement is possible for the first time. In fact, our current estimate is that it is possible that the TPC will achieve close to $0.5 \%$ as shown in table 1. This gives the experiment some room for unforeseen problems, and raises the confidence level of achieving a true sub-percent measurement. All of the systematic uncertainties will need to be addressed to ensure they are negligible. The systematic uncertainty evaluations will require at least an order of magnitude more data than will be used in what might be considered the cross section measurement data set. The uncertainty analyses will require a number of special experimental configurations and run conditions, which is addressed in the 


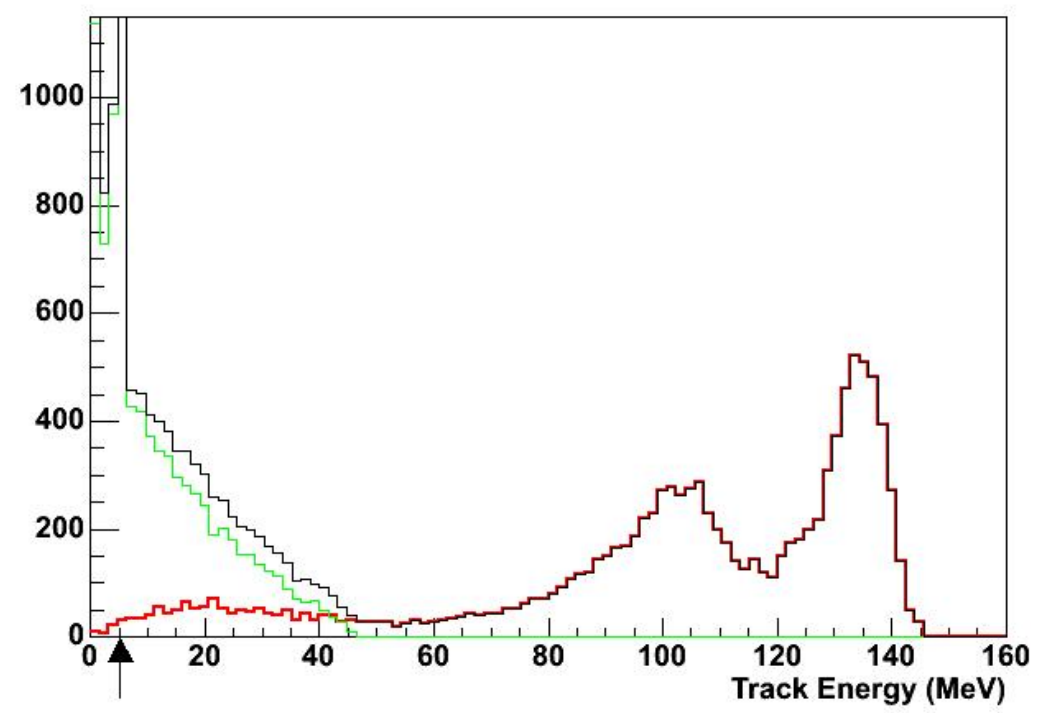

Figure 3: Shown here is the track energy distribution for a fission experiment simulation. The red distribution is the doublehumped fission energy spectrum with a low energy tail that results from scattering of some of the fission products in the target material and backing. The green distribution is from lighter particles, such as alphas and proton scatters. The combined distribution is shown in black and is what experimentalists will record. In order to count the total number of fission events, an assumption has to be made about the fission distribution under the alpha contribution. This assumption leads to an uncertainty of at least $1 \%$ on the total count and is highly dependent on the thickness of the target.

following section.

\section{$3 \quad$ Experimental Configurations}

In order to address the systematic errors enumerated and discussed above, a number of experimental configurations will be required to look at each error and quantify it. The statistical errors for each measurement need to be small enough to assess the systematic error under investigation, and a typical calculation of the number of neutrons needed for a configuration is shown next.

\subsection{Example Calculation of Required Number of Neutrons}

For this example calculation we pick some typical values, 1 barn cross section, $100 \mu \mathrm{g} / \mathrm{cm}^{2}$ target thickness, and a statistical error of $0.2 \%$. First we calculate the number of fissions from a given number of neutrons:

$$
N_{f}=100 \mu \mathrm{g} / \mathrm{cm}^{2} \cdot 1 \times 10^{-24} \mathrm{~cm}^{2} \cdot \frac{6 \times 10^{23}}{239 \times 10^{6} \mu \mathrm{g}} \cdot N_{n}
$$




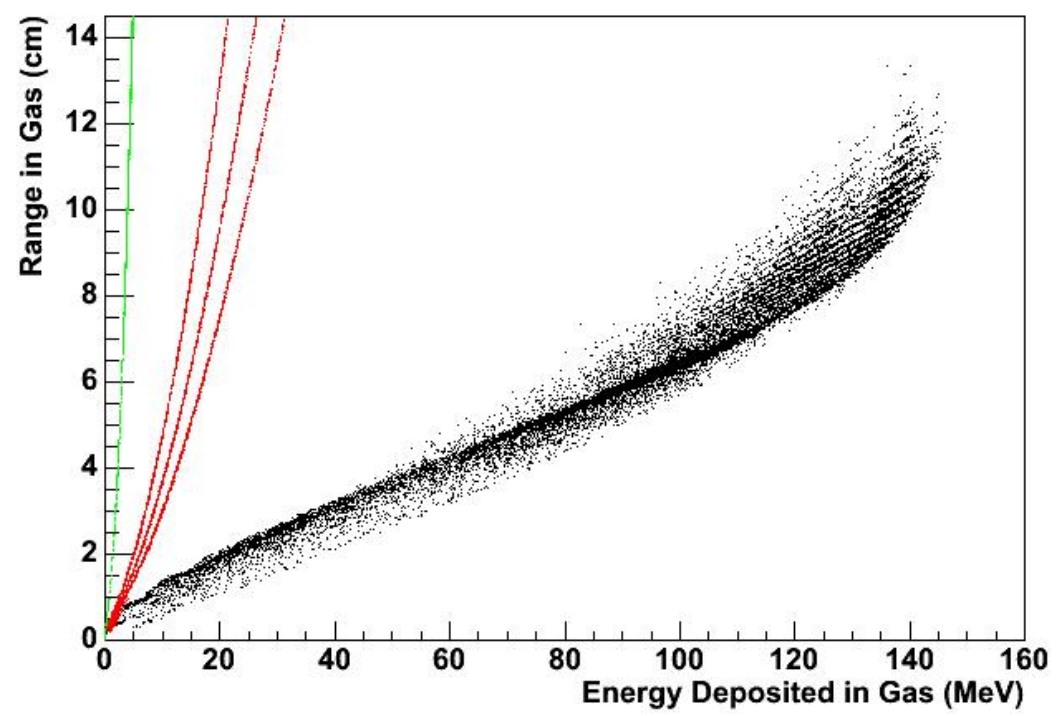

Figure 4: Shown here is the track length versus the track energy for the same events depicted in the previous figure. The lighter species are easily identifiable and easy to distinguish from fission fragments.

which reduces to:

$$
N_{f}=2.5 \times 10^{-7} N_{n}
$$

Where $N_{f}$ is the number of fissions and $N_{n}$ is the number of neutrons that land on target.

In order to get $0.2 \%$ statistical uncertainty we need $1 /(0.002)^{2}=2.5 \times 10^{5}$ fissions. To get the number of neutrons we just use the equation 2 .

$$
N_{n}=2.5 \times 10^{5} / 2.5 \times 10^{-7}=10^{12}
$$

So the nominal configuration will need about a trillion neutrons to reduce the systematic error below the systematic error under study. This is easily scaled by changes in the target thickness for example if the the target is only half as thick then twice the number of neutrons are needed, and the same for looking at different energy regions where the cross section is different than one barn.

The time to collect enough neutrons for $0.2 \%$ statistical uncertainty is calculated below and is about 18 days at LANSCE in $100 \mathrm{~Hz}$ operation. This should be compared with the time to design, construct, setup and analyze the data from a configuration. Depending on the complexity of the setup, these steps can take from days to months. For this reason it is generally more efficient to take the full amount of statistics needed in one configuration before moving to the next. Switching rapidly between configurations not only suffers from the time overhead of the work to switch, but also does not match the typical beam/maintenance cycle at LANSCE which has in the past been about 3 weeks on and 1 week off. 


\subsection{Configurations}

It is not possible to be exactly certain what configurations will be needed to address all of the systematic errors effectively. This is primarily for two reasons. The first is that this has never been done before, so we can only guess from prior experience in similar experiments as to the problems we will face and the time it will take to overcome them. The second is that the path of work depends significantly on what is learned in previous steps. This again makes the estimate difficult and we again have to rely on estimations based on experience of similar situations, and consider the experiment as a whole where some items will be more difficult than expected and some will be less. In addition to both of these issues, one also has to estimate the ability to get beam when needed, how and when funding will arrive, the skill of the workforce we can attract to the project, and the amount of management and bureaucratic overhead. Although we have looked at the timeline of this project from a number of angles and spent considerable time estimating the time needed to accomplish this project, it is difficult to fully enumerate and summarize all of the estimates and guesses developed over the years that go into the project, but I will layout a couple of simple estimates that illustrate how the work could proceed.

A very simple estimate would be to take the nominal number of neutrons needed for one configuration and multiply by the number of systematic errors and multiply by an factor that represents the amount of data that turns out to be useful after cuts. There are 13 systematic errors listed in this report. If we assume $1 / 2$ of the data is useful, that results in the need of about 26 trillion neutrons.

Another more detailed estimate would be to make some guesses as to needed configurations for specific systematic errors. For example, the Particle ID Degradation error is caused by the finite thickness of the target. It would be natural to assume that a few different target thicknesses are needed to assess the effect of the target thickness and very that we can measure and correct for energy loss in the target. This is also an example of how one step effects following steps. If it turns out that we can correct for energy loss in a thicker target, that could reduce the amount of beam time need in the study of other systematic errors. Another example is beam and target non-uniformity. This could be assessed by different target configurations. An example of configurations is shown in table 2. The total number is not that different than the simple calculation.

The total time to run and digest a configuration is not completely limited by beam time. There is considerable time consumed by the setup of the configuration, and then the analysis of that data set. In addition, in some cases the full statistics are needed before the systematic can be studied. For example the Particle ID degradation has a target of $0.2 \%$ uncertainty. This would be very difficult to assess without comparable statistics. The configuration changes also have to be fit into the schedule of the accelerator providing beam. For example, we would like to make the configuration changes during the maintenance periods when there is no beam. This provides the best use of the beam.

\section{Beam Estimates}

This section discusses the neutron beam and the time estimates based on what is expected from the beam facilities. 


\begin{tabular}{|c|c|c|c|}
\hline Target & Configuration & Reason & Neutrons $\left(\times 10^{12}\right)$ \\
\hline \multirow{9}{*}{$\begin{array}{l}\text { Solid Al, } 235 / 239 \\
200 \mathrm{UV} / \mathrm{cm} \text { on each } \\
\text { side }\end{array}$} & $\begin{array}{l}\text { Nominal settings - low } \\
\mathrm{E}\end{array}$ & $\begin{array}{l}\text { Nominal Measure- } \\
\text { ment }\end{array}$ & 0.5 \\
\hline & $\begin{array}{l}\text { Flip TPC around } \\
\text { nominal - lowE }\end{array}$ & $\begin{array}{l}\text { Beam } \\
\text { Spread/Scattering }\end{array}$ & 0.5 \\
\hline & Move to side of beam & Room return & .25 \\
\hline & 3.6us running & wrap around & 0.5 (1.0 eff $)$ \\
\hline & 7.2us running & wrap around & 0.25 (1.0 eff $)$ \\
\hline & notch filter & wrap around & 0.5 \\
\hline & low pressure & $\begin{array}{l}\text { high energy loss in tar- } \\
\text { get }\end{array}$ & 0.25 \\
\hline & Gain Change & Device Study & 0.25 \\
\hline & $\begin{array}{ll}\text { Nominal } & \text { Settings } \\
\text {-high E } & \end{array}$ & $\begin{array}{l}\text { get enough stats high } \\
\text { E }\end{array}$ & 1.0 \\
\hline \multirow[t]{2}{*}{$\begin{array}{l}\text { Solid Al, 235/239 } \\
50 \mathrm{UT} / \mathrm{cm} \text { each side }\end{array}$} & $\begin{array}{l}\text { Nominal settings - low } \\
\mathrm{E}\end{array}$ & Energy loss in deposit & 0.25 \\
\hline & Low pressure & $\begin{array}{l}\text { high energy loss in tar- } \\
\text { get }\end{array}$ & 0.25 \\
\hline \multirow[t]{2}{*}{$\begin{array}{l}\text { 100ug carbon, } \\
235 / 239 \text { pie } 100 u g\end{array}$} & $\begin{array}{l}\text { Nominal settings - low } \\
\mathrm{E}\end{array}$ & $\begin{array}{l}\text { Two fragment mea- } \\
\text { suremet }\end{array}$ & 1.0 \\
\hline & Low pressure & $\begin{array}{l}\text { high energy loss in tar- } \\
\text { get }\end{array}$ & 0.5 \\
\hline \multirow[t]{2}{*}{$\begin{array}{l}30 \text { ug carbon, } 235 / 239 \\
\text { pie } 100 \text { ug }\end{array}$} & $\begin{array}{l}\text { Nominal settings - low } \\
\mathrm{E}\end{array}$ & Thin backing & 1.0 \\
\hline & Low pressure & $\begin{array}{l}\text { high energy loss in tar- } \\
\text { get }\end{array}$ & 0.5 \\
\hline \multirow[t]{2}{*}{$\begin{array}{l}\text { 30ug carbon, } 235 / 239 \\
\text { pie } 20 u g\end{array}$} & $\begin{array}{l}\text { Nominal settings - low } \\
\mathrm{E}\end{array}$ & $\begin{array}{l}\text { Very thin tar- } \\
\text { get/backing }\end{array}$ & 2.5 \\
\hline & Low pressure & $\begin{array}{l}\text { high energy loss in tar- } \\
\text { get }\end{array}$ & 1.25 \\
\hline Total & & & 12.5 \\
\hline
\end{tabular}

Table 2: Example of a possible campaign of configuration changes for the fission TPC

\subsection{LANSCE}

The beam at LANSCE has been measured and is plotted in figure 4 of PRC79,014613(2009). This flux was measured at $40 \mathrm{~Hz}$ and is about $10^{6} \mathrm{n} / \mathrm{s} / \mathrm{cm}^{2} / \mathrm{MeV}$ from about $100 \mathrm{keV}$ up to about $2 \mathrm{MeV}$. It then drops to about $10^{5} \mathrm{n} / \mathrm{s} / \mathrm{cm}^{2} / \mathrm{MeV}$ at $7 \mathrm{MeV}$ and about $4 \times 10^{4} \mathrm{n} / \mathrm{s} / \mathrm{cm}^{2} / \mathrm{MeV}$ at $14 \mathrm{MeV}$.

At $40 \mathrm{~Hz}$ operation and $1.8 \mathrm{~cm}$ diameter target (the target is $2.0 \mathrm{~cm}$ in diameter, assume the target data near the edge is unusable) then the number of neutrons per $24 \mathrm{hr}$ day below $2 \mathrm{MeV}$ is about 


$$
10^{6} / 10 \cdot \pi(0.9)^{2} \cdot 86400=2.2 \times 10^{10} \text { neutrons } / \text { day } / 100 k e V
$$

Assume statistical precision of $0.2 \%$, we need about a trillion neutrons (equation 3 ). This would take 45 days at $40 \mathrm{~Hz}$ and 18 days at $100 \mathrm{~Hz}$.

At $7 \mathrm{MeV}$ the flux drops off by about 10 increasing the beam time to 450 days and 180 days correspondingly.

It was originally assumed that LANSCE would deliver $100 \mathrm{~Hz}$ beam by this time. It now looks like the best projection is that we will see $40 \mathrm{~Hz}$ for the next two cycles and then $100 \mathrm{~Hz}$ for the 2 after that. The beam generally runs for about 6 months and is on for 3 weeks and then off 1 week for maintenance. This provides about 135 days per year, or about 3 trillion neutrons per year for the $40 \mathrm{~Hz}$ running and 7.5 trillion a year at $100 \mathrm{~Hz}$. That makes a total of 21 trillion over then next 4 years if the $100 \mathrm{~Hz}$ operation is started the year after next. From our current understanding, this appears to be sufficient to carry out the program.

\subsection{Fixed energy source}

The original plan was to install a high flux neutron source in the basement of building 194 at LLNL. This did not happen, and the result will be a lower confidence on the cross sections as we can not perform as many cross checks. We are attempting to mitigate this with running at other facilities and we are currently working on that plan. For the purpose of this report, we make some estimates of what the source that was planned at LLNL would do.

With the high intensity source and a $1 \mathrm{~m}$ stand off for collimation and time of flight, we may get of order $10^{5} \mathrm{n} / \mathrm{s} / \mathrm{cm}^{2}$. Assuming a $1.8 \mathrm{~cm}$ target, this works out to about the same number of neutrons/day as LANSCE but only at one energy.

The flux is not as energy dependent so the time to collect $0.2 \%$ statistics at $2 \mathrm{MeV}$ or $7 \mathrm{MeV}$ is about 45 days.

\section{Conclusion}

The fission TPC project will take about 4 years to make the precision ${ }^{239} \mathrm{Pu}$ cross section measurement. This estimate relies on $100 \mathrm{~Hz}$ operation at LANSCE beginning in FY13 and a significant amount of time (12-18months) at a fixed energy source. The estimate also depends on details that are difficult to a priori estimate because this is the first experiment of its kind. Given that the major experimental concern is addressing systematic uncertainties at the sub-percent level, measurements that address these issues dominate our experimental run time. The actual optimized "production" experiments are a relatively small fraction of our experimental plan. 